\title{
Bitschwankung in den Laufwerken
}

\author{
György Györök
}

Zur Steigerung der Speicherkapazität von Floppys unter Beibehaltung des vorgegebenen Raumbedarfs gibt es zwei Möglichkeiten: Erhöhung der Zahl der Schreibspuren, Erhöhung der Schreibdichte.

Als Lösung des ersten Verfahrens bietet sich die Erhöhung der Übersetzung an (angefangen von der Drehbewegung des Motors bis hin zur fastlinearen Bewegung des Kopfes). Dies kann aber wegen der Histerese nicht ins Unendliche gesteigert werden. Die Übersetzung in der bisherigen Größenordnung bleibt und es wird das Verfahren der Vervielfachung der Schritte des Schrittmotors, die sogenannte Mikroschrittbetriebsart, angewendet.

Wegen der Reibung der mechanischen Teile und des zulässigen Erregerstromes in den Spulen des Schrittmotors kann die Mikroschrittbetriebsart die Zugriffszeit nicht unbegrenzt erhöhen. Dieses Verfahren wird bei den meisten der verwendeten Floppytypen angewendet. Bleibt somit das in der zweiten Möglichkeit vorgeschlagene Verfahren, bei dem die Länge der bescheidenen Magnetzone verringert bzw. die Schreibdichte erhöht wird. Zur Erhöhung der Schreibdichte sind auch zwei Modalitäten möglich: physikalische Verdichtung und/ oder die Verdichtung durch Kodierung.

\section{Wie weit kann die Schreibdichte physikalisch erhöht werden? Die Antwort darauf kann in Kurzform folgen- dermaßen definiert werden, dass dies fast ausschließlich vom Lese/Schreibkopf (Luftspalt, Streuung in XY-Rich- tung, Abstand zwischen Kopf und Platte usw.) und von der Qualität des Magnetdatenträgers (Koerzivität und Remanent der Magnetschicht usw.) abhängt. Dies hängt natürlich wiederum mit der Fertigungs- und Montage- technologie zusammen. Die Erhöhung der Schreibdichte mittels Kodierung ist als Grundlage selbstverständlich. Die am Interface ankommenden Daten werden auf der Magnetplatte mit der RZ-Kodierung erfasst. Innerhalb dessen sind MFM, M²FM und RLL (Run Length Limit) am verbreitetsten.}

Die MFM-Variante ist eine solche Kodierung, die mit fiktiven Zonen arbeitet. Wenn wir schreiben, so muss der Impuls in die Mitte der Bitzelle gefuihrt werden und bei 0 an deren Anfang (Abb. 1).

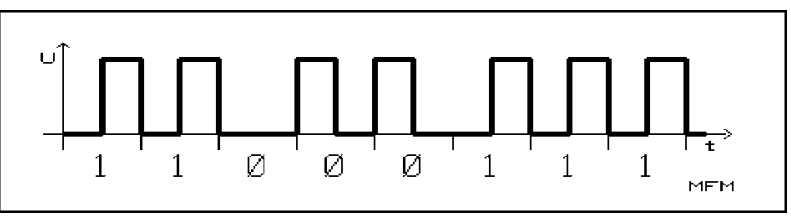

Abb. 1: Die MFM-Kodierung
Bei diesem Algorithmus entsteht dann ein Problem, wenn wir nach der 1 die 0 schreiben wollen, da sich die Impulse dann „berühren“ würden. Wir schreiben, wenn zwischen zwei Einsen eine 0 liegt, an diese Stelle keinen Impuls. Wenn dagegen mehrere Nullen nacheinander folgen, wird an den Anfang der ersten Zelle nicht aber zu allen weiteren ein Impuls geschrieben. Dies hat eine Bedeutung für die Datenseparierung nach dem Lesen.

$M^{2} F M$ ermöglicht gegenüber MFM bei durchschnittlichem Datenmuster eine Erhöhung der Datenspeicherung auf das 1,1- bis 1,2-fache. Die Codierung stimmt mit der von MFM überein, weicht aber davon ab, wenn wir mehrere Nullen schreiben. Dann wird zu den ersten beiden kein, aber zu allen folgenden ein Impuls geschrieben (Abb. 2).

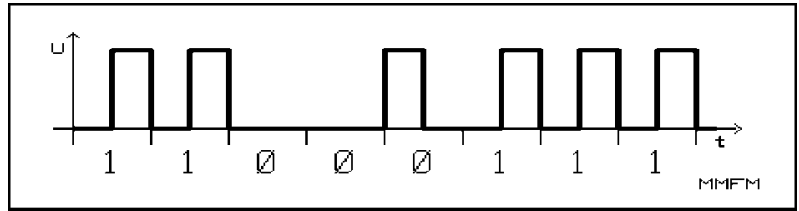

Abb. 2: Die $M^{2} F M$ Kodierung

Die RLL-Verdichtungstechnik steigert die Speicherkapazität um $50 \%$ gegenüiber der MFM-Technik. Bei der FMTechnik beträgt der Wert der minimalen Lauflänge 0 (es ist möglich, dass es zwischen den Impulsen keine Pausen gibt) und der maximalen Lauflänge 1 (nach der Pause ist immer ein Taktsignal). Auf diese Weise ist für FM die beschränkte Lauflänge 0,1 oder kurz 0,1 RLL. Ähnlich wie bei MFM ist immer wenigstens eine Pause zwischen den Impulsen, aber nicht mehr als 3, d. h. 1,3 RLL. Im Allgemeinen bestimmt der Minimalwert, wie dicht die Daten auf der Platte angeordnet werden können, und der Maximalwert die Taktgenauigkeit des Steuerschaltkreises, da die Taktsignale auch dann erzeugt werden müssen, wenn eine Pause ist. Weiterhin wird dadurch der örtlichen Streuung des Bits ein strenges Kriterium gesetzt.

Das unter RLL bekannte Kodierungsverfahren ist ein 2,7 RLL (Abb. 3). Dies verwendet wesentlich komplexere Kodierungsregeln zur Beschreibung der Impulsfolgen

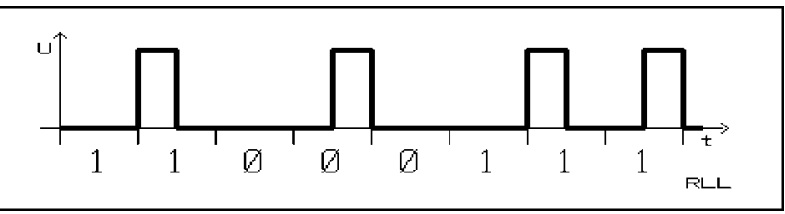

Abb. 3: A 2,7 RLL 
der Bits unter Ausnutzung des Wertes des vorhergehenden Datenbits. Das Grundprinzip ist aber gleich: Es werden weniger Impulse verwendet, aber eine wesentlich genauere Taktung gefordert. Die Länge der Formation hängt von den ursprünglichen Datenbits ab, aber die Aufteilung der Impulse garantiert die oben genannte 2,7 Maximal- und Minimallauflänge.

Natürlich existieren noch weitere Verdichtungsverfahren wie das ARLL [Advanced RLL] und das ERLL [Enhanced RLL], welche die Datendichte um $100 \%$ steigern. Bei ARLL- und ERLL-Systemen kommen die Genauigkeitskriterien noch verstärkt zur Geltung (Abb. 4).

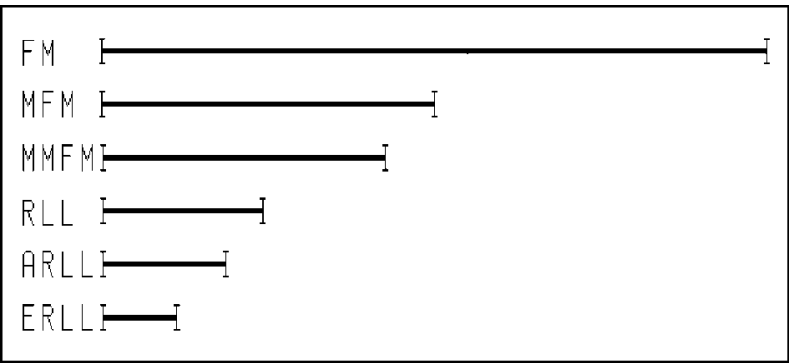

Abb. 4: Vergleich der verschiedenen Kodierungen

Was schränkt die Wahl der für einzelne Speichermedien verwendeten Kodierungsalgorithmen ein? Wie wir zuvor sahen, ist nicht der magnetische Wechsel bzw. dessen Fehlen, sondern eher der Ort des Wechsels in der darstellenden Kodierung für die immer größer werdenden Informationen (je Einheit) verantwortlich. Wenn wir also bei den einzelnen Magnetspeicherplatten die Beständigkeit des Ortes des Magnetwechsels sichern können, so können wir Kodierungen verwenden, die eine immer größere Verdichtung erreichen.

Was verursacht die Veränderung des Bitortes? Etwas spöttisch könnte man sagen, was diese Erscheinung nicht verursacht. Auf jeden Fall sind die Bitortschwankungen in zwei Gruppen einzuteilen: determinitstische, stochastische.

Das auf den Datenträger geschriebene Magnetisierungsmuster ist eine Folge von Zonen mit entgegengesetzter Ausrichtung. Solange die Felder sich unter dem Kopf befinden, können die durch die große Permeabilität des Kopfkernes ausgelösten, gegenseitig wirkenden Einfluisse vernachlässigt werden. Die benachbarten Magnetzonen beeinflussen sich gegenseitig, wenn sich die Zonengrenzverschiebungen vom Kopf entfernen, wobei dann die magnetische Feldstärke wächst und sich der gegenseitige Einfluss der Felder verstärkt. Deswegen wird der magnetische Wechsel (die Zonengrenze) länger und deren Ort verändert sich. Diese Erscheinung bezeichnen wir als Spitzenverschiebung (Bitschwankungen, Pick Shift).

Die Richtung und Größe der Zonengrenzverschiebung hängt von der Größe (Länge) der beiden benachbarten Felder ab. Die größere Feldstärke des längeren Feldes schwächt des kleinere Magnetfeld (siehe Abb. 5: PS2, ... PS6), weswegen sich die Spur mit der Null-Feldstärke in
Richtung der kürzeren Zone bewegt (Superposition). Wenn Felder gleicher Größe einander folgen, bleiben die Zonengrenzen an ihrem Ort (Abb. 5: PS1).

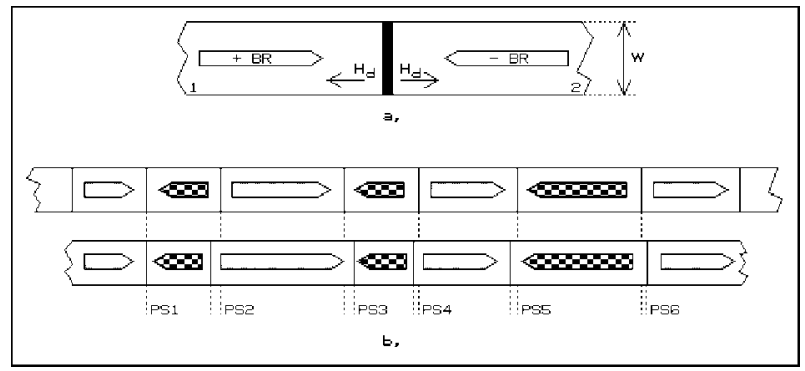

Abb. 5: Die Spitzeverschiebung

Die Erscheinung der Bitverschiebung wird durch einige Parameter trivial verstärkt, wie z. B. Magnetisierungstiefe der Datenträger, Wirkung der Luftspaltbreite, Wirkung des Echtzeitmagnetwechels, Bewegungsgeschwindigkeit des Datenträgers, Bahnbreite, Wirkung der Schräge des Luftspaltes.

1. Der im Luftspalt des Kopfes befindliche Magnetfluss ist die Summe des aus den elementaren Magnetteilchen stammenden Magnetflusses. Bei Vergrößerung der Entfernung zwischen Kopf und Datenträger wird durch die vom Luftspalt entfernteren Felder im Kopf auch Spannung induziert. Mit wachsender Entfernung sinkt die induzierte Spannungsamplitude. Diese Erscheinung veranschaulichen die Kurven $a, b, c$ der Magnetisierungstiefen in der Abbildung 6 . Die Schichtdicke hat eine Wirkung auf das gelesene Signal.

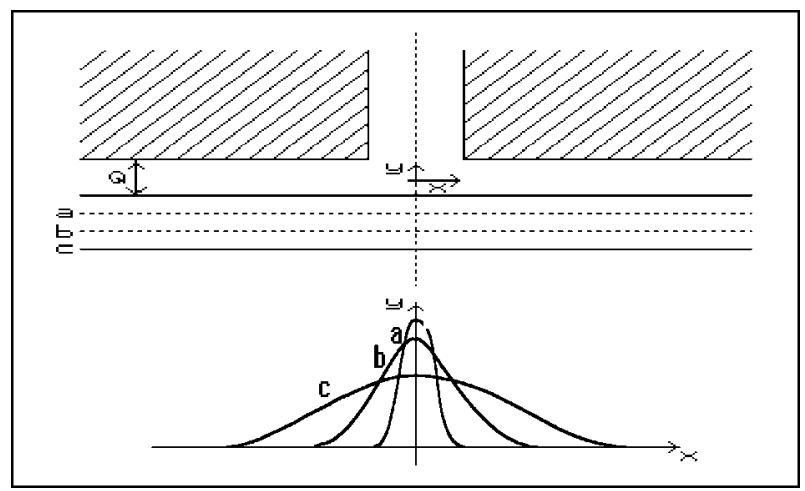

Abb. 6: Die Signalform an verschiedenen Kopf-Disk-Kontakten

2. Die Breite des Luftspaltes beim Lesen und Schreiben hat einen Einfluss auf das beschriebene Magnetisierungsmuster bzw. auf die Parameter des gelesenen Signals.

Es ist einzusehen, dass, wenn die Werte für Luftspaltbreite, Entfernung Kopf-Platte und Magnetisierungsschichtdicke auf dem kleinsten Wert gehalten werden, die Spitzenverschiebung ein Minimum ergibt.

3. Die Remanenzinduktion des beschriebenen Magnetisierungsmusters beim Schreiben ändert sich nicht sprunghaft, sondern stetig. Die Amplitude des gelesenen Signals sinkt und die Breite wächst entsprechend der Zunahme der Zeit für den Wechsels. 
4. Es ist auch einzusehen, dass die induzierte Spannung in der Spule des Lesekopfes eine lineare Funktion der Geschwindigkeit des Datenträgers ist. Anderseits ist bekannt, dass die Breite des gelesenen Signals im umgekehrten Verhältnis zur Geschwindigkeit steht. Auch in diesem Fall ist also die Bestimmung des optimalen Wertes das Ziel.

5. Die Amplitude des gelesenen Signals steht im direkten Verhältnis zur Breite der Bahn. Dies kann aber nicht beliebig erhöht werden, da dazu eine große Oberfläche des Datenträgers notwendig und somit die Ausnutzung des Datenträgers gering wäre.

6. Wegen verschiedener mechanischer und fertigungstechnologischer Probleme ist der Luftspalt des Kopfes selten vertikal zur Bewegungsrichtung des Datenträgers (Abb. 7). Diese Erscheinung ist vollständig äquivalent mit der Wirkung des Echtzeitmagnetisierungswechsels.

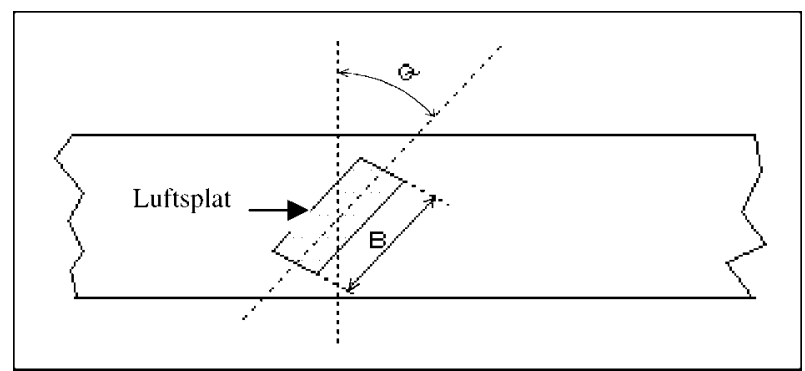

Abb. 7: Die Signalform an verschiedenen Kopf-Disk-Kontakten

Die Größe der Spitzenverschiebung ist ein wichtiger Parameter. Beim Schreiben soll auf die Präkompensation (Vorkorrektur) des zu schreibenden Datenwertes geachtet werden, damit beim Lesen mit dem Signalverarbeitungsschaltkreis die Daten entsprechend behandelt werden können. Ein solcher Schaltkreis kann durch Korrektur der deterministischen Verschiebung ausgebildet werden.

Die stochastische Bitverschiebung hängt ab von den obigen Parametern, und selbstverständlich hängen davon auch ab:

1. die Schwankungen der Winkelgeschwindigkeit des Antriebsmotors (der angetriebenen Platte bzw. des Plattenstapels). Für die heute üblichen Magnetplattenspeicher werden als Antriebsmotor ausschließlich Direktmotoren verwendet. Dessen Winkelgeschwindigkeitsschwankungen sind klein. Sie halten eine genaue konstante Drehzahl. Infolge der Winkelgeschwindigkeitsschwankungen, die sich aus der elektronischen Kommutation ergeben, kann Jitter auftreten. Dem kann man nur durch eine größere Zahl von Kommutatoren bzw. einen integrierenden Schwungrad entgegenwirken.

2. die sich aus dem Signalweg (Schaltkreis) ergebenden stochastischen Schwankungen. Die typische Signalart des Lesestromkreises ist in Abbildung 8 zu sehen. Das aus dem Kopf ankommende Signal gelangt in die Verstärkerleistung von $40 \mathrm{~dB}$. An dessen Ausgang befindet sichern Tiefpassfilter. Mit diesem Filter werden einesteils

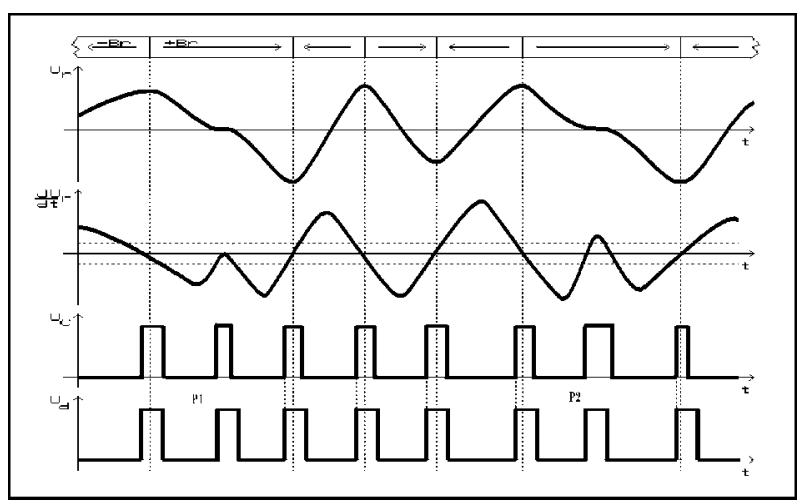

Abb. 8: Die verschiedenen Arten der Lesesignale im Leseverstärker

die Hochfrequenzstörungen herausgefiltert und andererseits die Laufzeitkorektur durchgefuihrt.

\section{Literatur}

Németh, Gábor; Mozgó mágneses tárolók, BME, 1977

Györök, György; Perifériák KKMF-1175, Foiskolai jegyzet $1996,1999,2002$

Györök, György; Application of a system identification method for hard disk drives

UNITECH'01 2001, International Scientific Conference, November 22-23, Gabrovo Bulgaria 2001

\section{Autor}

\section{Dr. György Györök}

Technische Hochschule Budapest

Kandó Kálmán Fakultät, Institut für Computertechnik

Budai út 45, 8000 Székesfehérvár, Ungarn

Tel. + 36 (22) 316-260

Fax +36 (22) 312-337

E-Mail: gyorok.gyorgy@szgti.bmf.hu 